Review

\title{
Role of Cytokines and Transcription Factors in Periodontitis: A Review of Cellular and Molecular Mechanisms
}

\author{
${ }^{1}$ Anne Carolina Eleutério Leite, ${ }^{1}$ Valéria Martins de Araújo Carneiro, ${ }^{2}$ Júlia Faria Nunes, \\ ${ }^{2}$ André Cruz de Sousa, ${ }^{3}$ Maria Imaculada Muniz-Junqueira and ${ }^{1}$ Maria do Carmo Machado Guimarães \\ ${ }^{1}$ Department of Dentistry, Periodontics Division, University of Brasilia, Brazil \\ ${ }^{2}$ Department of Dentistry, Periodontics Division, Catholic University of Brasilia, Brazil \\ ${ }^{3}$ Faculty of Medicine, Pathology, Cellular Immunology Laboratory, University of Brasilia, Brazil
}

Article history

Received: 29-04-2015

Revised: 09-10-2015

Accepted: 19-11-2015

Corresponding Author: Anne Carolina Eleutério Leite, Department of Dentistry, Periodontics Division, University of Brasília, Brazil

Tel: +55 (61) 3107-3300

Email: annecarolinaleite@gmail.com

\begin{abstract}
Periodontal Diseases (PD) are characterized by pathological manifestation of host immune response to bacterial infection at the tooth/gingival interface. Evidences points periodontitis as a risk factor for pathological systemic conditions, such as, cardiovascular diseases and diabetes. The identification of host factors that determine their susceptibility to immune subversion can provide useful information in the pathogenesis of periodontitis. Protective acute inflammatory response fails to remove inflammatory cells, especially neutrophils, evolving to chronic, destructive and pathological lesions. T and B cells actively participate in pathogenesis of the disease. CD4+ naive T cells are activated by antigenic stimulation and differentiate into subpopulations of distinct effector cells, characterized by its specific cytokine production profiles and functions. In periodontal infection, activated Th17 and regulatory T lymphocytes (Tregs) play antagonistic roles as effector and suppressor cells, respectively. In presence of Tregs, there is a decrease in the levels of pro-inflammatory cytokines, such as, Interferon gamma (IFN- $\gamma$ ), Interleukin (IL) -17, Tumor Necrosis Factor (TNF) and IL-1 $\beta$, at sites of disease. Absence of Tregs may cause a variety of disorders, such as, periodontitis. The RANKL/RANK system and Osteoprotegerin (OPG) are modulators of bone resorption in periodontitis. The balance between periodontal bone resorption by osteoclasts and bone formation by osteoblasts controls bone mass. RANKL induces osteoclast differentiation and maturation and OPG inhibits RANK/RANKL interaction and prevents bone resorption. RANKL mRNA and the RANKL/OPG mRNA ratio were enhanced in chronic periodontitis. Furthermore, the role of NF-kB, FoxP3 and T-bet transcription factors were explored. Therapies for periodontitis involving cellular and molecular biology are not specific and have many side effects. Current therapies to successfully control the PD reduces clinical signs of inflammation at local sites of the disease; however, new treatments for periodontitis should address the contribution of immune cells in bone resorption, particularly in the natural course of the disease, considering its periods of remission and progression.
\end{abstract}

Keywords: Transforming Growth Factor Beta, T Helper Cell, Periodontal Diseases, RANKL/RANK

\section{Introduction}

Periodontal Diseases (PD) are characterized by pathological manifestation of host immune response to bacterial infection at the tooth/gingival interface. These are mainly caused by Gram-negative bacteria, including Porphyromonas gingivalis, Prevotella intermedia, Aggregatibacter actinomycetemcomitans and Tannerella forsythia. Differences in individual response can be explained by the combination of factors that confer upon 
them a complex nature. Among these factors, susceptibility becomes prominent, with special attribution given to genetic polymorphisms, environmental factors and pathogen virulence factors (Ohlrich et al., 2009; Sanz and Winkelhoff, 2011). Individuals affected by the disease share common polymorphisms in specific genes that are important for regulation of inflammatory response (D'Aiuto et al., 2004d; Fitzsimmons et al., 2010). However, determinants for susceptibility to periodontitis remain still unclear (Linden et al., 2013).

Severe periodontitis affects up to $15 \%$ of most populations (Papapanou, 1999). In Brazil, the most recent National Survey of Oral Health reported a prevalence of $19.4 \%$ and the most severe forms of periodontal disease was more significant in adults between 35-44 years of age (Leite et al., 2014).

Periodontitis comprises chronic forms of periodontal disease, which are the result of a polymicrobial infection and are characterized by loss of collagen fibers and insertion in the cementum surface (mineralized tissue that covers the root surface), apical migration of junctional epithelium (epithelium continuous with the oral epithelium, which promotes the insertion of the gum to the tooth), periodontal pocket formation (cementum surface devoid of periodontal fibers) and alveolar bone reabsorption. Such damages impair functions of periodontal tissues and may result in tooth loss (Sanz and Winkelhoff, 2011).

There are more than 700 bacterial species in periodontal pockets (Aas et al., 2005) and a combination of aerobic and anaerobic microbiota is typically seen in infection. Substantial tissue destruction in patients with severe periodontitis is characterized, in many cases, by deep periodontal pockets around many or all teeth. The aggregate epithelial lesions are equal in size to an ulcerated wound with an area of 8 to $20 \mathrm{~cm}^{2}$, in accordance with clinical estimations (Slade et al., 2000). Thus, the chronic and cyclical nature of periodontal disease provides an opportunity for continuous hematogenous dissemination of periodontal pathogens, bacterial antigens and inflammatory mediators (Offenbacher et al., 2005; Linden et al., 2013).

Since the beginning of the 1990s, evidence has pointed to periodontitis as a risk factor for systemic conditions, such as, Cardiovascular Disease (CVD), adverse outcomes of pregnancy, diabetes, rheumatoid arthritis and lung disease (Williams and Offenbacher, 2000; Hajishengallis, 2014).

In healthy gingiva, the sulcular epithelium and local innate immunity act as a natural barrier that prevents bacterial penetration. However, in PD, the epithelium of ulcerated and inflamed gingiva is vulnerable to bacteria and creates a gateway to subsequent tissues (Linden et al., 2013). Bacteria and bacterial antigens that are systemically dispersed cause significant systemic inflammation. Leukocytes, epithelial and endothelial cells and hepatocytes respond to virulence factors with secretion of proinflammatory immune mediators, as cytokines, chemokines (e.g., Monocyte Chemoattractant Protein-1 (MCP-1) and Macrophage Inflammatory Protein-1alpha (MIP-1alpha)), C-Reactive Protein (CRP), an acute phase protein (Gemmell et al., 2001; Maekawa et al., 2011). With continued exposure, soluble antigens react with specific circulating antibodies to form immune complexes that amplify inflammation in places where there are immune complex deposition. Similarly, pro-inflammatory mediators, such as Interleukin (IL)-1 $\beta$, IL-6, Tumor Necrosis Factor alpha (TNF- $\alpha)$, Prostaglandin E2 ( $\left.\mathrm{PGE}_{2}\right)$, among others, locally produced in inflamed gingival tissues can spread into the systemic circulation. The pro-inflammatory cytokines in circulation induce leukocytosis and acute phase proteins production (e.g., CRP, fibrinogen and serum amyloid A) (Linden et al., 2013; Hajishengallis, 2014). In this context, the increase in the number of White Blood Cells (WBC) is associated with the increased risk of coronary heart disease, CVD, atherosclerosis, thrombosis and myocardial ischemia. This increase may be caused by the inflammatory nature of chronic infections, such as, periodontitis (Loo et al., 2012).

It has been shown that the $\mathrm{T} C \mathrm{CD} 4+\mathrm{CD} 25+$ regulatory cells (Tregs) that express transcription factor FoxP3 may prevent the overreactive adaptive immune response associated with PD (de Brito Bezerra et al., 2012; Kobayashi et al., 2011). Tregs may reduce Interferon gamma (IFN- $\gamma$ ), IL-17, TNF and IL-1 $\beta$ levels at periodontal disease sites (Glowacki et al., 2013). IL-10, Transforming Growth Factor beta (TGF- $\beta$ ) and Cytotoxic T-Lymphocyte-Associated protein 4 (CTLA4) are produced by Tregs to control inflammation, slowing progression to periodontal disease (Linden et al., 2013). TGF- $\beta$ has also been studied as a form of treatment, because it may heal and regenerate periodontal bone (Glowacki et al., 2013).

This review aimed to report the cellular and molecular mechanisms involved in periodontal inflammation and alveolar bone loss, as well as the systemic effects in periodontitis.

\section{Classification of Periodontal Diseases and Microbial Associations}

In current clinical practice, two types of periodontitis are recognized: Chronic Periodontitis (CP) and Aggressive Periodontitis (AgP), as classified by the Current Classification of PD by the American Academy of Periodontology (AAP, 1999). CP has higher prevalence in adults, but can also occur in children and adolescents. In CP, there is correlation between the severity of bone destruction and the quantity of dental 
biofilm and calculus, in addition to the moderate growth rate in the majority of cases. Considering the extension of lesions, CP is classified as localized when less than $30 \%$ of dental sites (mesiobuccal, buccal, distobuccal, mesiolingual, lingual and distolingual) are affected and as generalized when it exceeds this limit. Whereas, AgP is characterized by the rapid loss of clinical attachment and alveolar bone. The following characteristics are considered for diagnosis of AgP: No contribution of systemic diseases and familial aggregation. According to the AAP, the diagnosis is based on clinical data, X-rays and clinical history. Localized Aggressive Periodontitis $(\mathrm{LAgP})$ is characterized by destruction of the periodontal tissues in the first molar/incisor with interproximal insertion loss in at least two permanent teeth, one of which is the first molar and involving no more than two teeth beyond the first molars and incisors. It is usually detected and diagnosed during puberty in systemically healthy individuals. It also has a strong serum antibody response to infectious agents. Generalized Aggressive Periodontitis (GAgP) usually affects individuals under age 30 , but can affect older individuals. GAgP is characterized as generalized when the loss of interproximal insertion affects at least three permanent teeth, in addition to the first molars and incisors. Moreover, GAgP progresses in alternating periods of activity and quiescence. In some period of activity, pronounced destruction of tooth insertion, alveolar bone destruction and insufficient response of serum antibodies to infectious agents has been observed (Armitage, 1999; Armitage and Cullinan, 2010).

Furthermore, it was recently suggested that $\mathrm{CP}$ is a polygenic disease, in which multiple genes contribute cumulatively to the overall disease risk (or protection) by influencing the host immune response and the composition and structure of the microbiota (Divaris et al., 2013). Nonetheless, monogenic forms of the disease are different, such as aggressive periodontitis, in young patients with leukocyte adhesion deficiency, in which a single gene (ITGB2; which encodes integrin $\beta 2$ ) invariably precipitates periodontal disease (Moutsopoulos et al., 2014).

A systematic review concluded that there is no evidence to support differences in the composition of subgingival microbiota between the clinical forms CP and AgP (Mombelli et al., 2002). Although no specific systemic marker was associated with the severity of periodontal destruction, it has been suggested that AgP may show a hyper-responsive systemic profile associated with genetic susceptibility (Cairo et al., 2010). It was also suggested that high titers of $A$. actinomycetemcomitans and $P$. gingivalis are suggestive of generalized and severe PD (Papapanou et al., 2004; Dye et al., 2005).
Recently, different serotypes of $P$. gingivalis have been associated with the induction of a distinct type of immune response, which suggests the role played by capsule during activation of dendritic cells (Hernández et al., 2011).

Inflammation is an important source of nutrients (especially for asaccharolytic bacteria) and thus exerts a powerful influence on the composition of periodontal microbiota, favoring species that can use tissue degradation products. Whereas, species that cannot benefit from these environmental changes may have disadvantage of fitness and thus are overcome. The selective growth of bacteria, which act as pathogenic bacteria, has the potential to trigger a self-feeding vicious cycle for subsequent tissue destruction and bacterial overgrowth (Hajishengallis, 2014).

Virulence factors such as cytotoxins, proteases and hemagglutinins, structural molecules of the bacteria, including Lipopolysaccharide (LPS) and Peptidoglycan (PGN), lipoproteins, bacterial DNA and double-stranded RNA interact with the host immune system. Most of these molecules have conserved motifs known as Pathogen-Associated Molecular Patterns (PAMPs), which are recognized by host cell receptors called Pattern Recognition Receptors (PRRs). The PRRs detect PAMPs in the environment and activate specific signaling pathways in host cells that initiate inflammatory responses. Virulence factors of bacteria, including PAMPs, are LPS, PGN, lipoteichoic acid (LTA), fimbriae, proteases, Heat Shock Proteins (HSPs), formyl-Methionyl-Leucyl-Phenylalanine (fMLP) and toxins. PRRs include Toll-Like Receptors (TLRs) and a variety of G-Protein Coupled Receptors (GPCRs). However, it should be noted that the most of these proposed interactions were only observed in vitro or in animal models (Hans and Hans, 2011; Linden et al., 2013).

\section{The Microbial Challenge: Cellular and Molecular Mechanisms in Periodontal Inflammation and Bone Loss}

During the last decade, increasing evidence supports the idea that changes in subpopulations of immune cells within the periodontal tissue may have an important impact on the clinical phenotype and progression of periodontal destruction. While Polymorphonuclear Leukocytes (PMNs) are the first line defense (innate immunity) in protecting the host from periodontal pathogens, the increase in the number of macrophages in subepithelial connective tissue has been involved in progression of PD. They are present in a greater number of active periodontal lesions compared to inactive sites. The phenotype of hypersecretory macrophages (hyperinflamatories) was hypothesized to account for the increased amount of IL-1 $\beta$ and TNF- $\alpha$ in periodontal 
sites of disease in progression when compared to inactive and healthy sites. In addition, the increased number of monocytes in periodontal tissues may promote the differentiation to bone-resorbing cells (osteoclasts) under bacterial stimulation, which could be responsible for bone loss that is observed in progressive periodontitis sites (Hernández et al., 2011).

The involvement of neutrophils in the pathogenesis of chronic diseases, such as periodontitis, may seem surprising, since they are usually associated with acute host responses to infection. However, neutrophils play an increasingly recognized role in chronic inflammatory diseases, such as, rheumatoid arthritis and psoriasis. In addition, it is uncertain whether the chronicity of periodontitis is a constant pathological process or a persistent series of brief acute bursts separated by periods of remission (Hajishengallis, 2014).

Alongside with the epithelial barrier, the action of phagocytes and the complement system, Natural Killer (NK) cells play an important role on the front line in the defense against antigens (Gemmell and Seymour, 1998).

TLRs in gingival epithelial cells are continuously stimulated, resulting in production of cytokines and defensins, which help to maintain oral health. If the epithelial barrier is broken, allowing the invasion of bacteria into the underlying connective tissue, the TLRs in other resident cells (neutrophils, macrophages and dendritic cells) and non-residents cells of the periodontium are activated. This leads to an excessive release of pro-inflammatory cytokines and other biological mediators, which can cause destruction of host tissues (Hans and Hans, 2011; Gonzalez et al., 2014).

After interacting with PAMPs, TLRs activate innate immune cells through intracellular signaling pathways. This innate immune response mediated by TLRs is also essential to activate adaptive immunity (Hans and Hans, 2011).

TLRs enhance expression of co-stimulatory molecules and production of cytokines and chemokines, which are critical for proliferation and differentiation of $\mathrm{T}$ cells (Hans and Hans, 2011). It is now known that immune cells release qualitatively and quantitatively different cytokines when stimulated by Gram positive and Gram-negative bacteria, indicating the specificity of these responses (Hans and Hans, 2011).

The genetic regulation which leads to the secretion of proinflammatory cytokines from a variety of cells depends on activation of NF- $\kappa B$. The NF- $\kappa B$ regulatory pathways are activated by PAMPs, through TLR pathway (Hanada et al., 2011; Linden et al., 2013; Guerrini and Takayanagi, 2014).

Recent research showed that in homeostatic balance between parasite and host, activation of TLRs by commensal bacteria is critical to maintain oral health. Gingival epithelial cells express TLRs 2, 3, 4, 5, 6 and 9 and recognize many microorganisms by these receptors. Therefore, signaling by TLRs limit the microbial invasion and prevent commensal microorganisms from breaching the epithelial barrier, thus maintaining gingival health (Hans and Hans, 2011).

Macrophages, phagocytic cells of the myeloid lineage, efficiently ingest particulate antigens and express Major Histocompatibility Complex molecules (MHC) class II that induce T cells activation. Dendritic cells also express MHC class II molecules and have costimulatory activity. Clearly, the innate and adaptive immune systems are coordinately involved in inflammatory response and destruction of tissues, although there is no complete understanding of the mechanism of many inflammatory diseases, including periodontitis, obesity, diabetes, rheumatoid arthritis and CVD (Linden et al., 2013).

It is known that when the acute periodontal lesion remains unresolved, bacterial antigens are processed and presented to the adaptive immune system by macrophages and dendritic cells. $\mathrm{T}$ lymphocytes recognize intracellular pathogens presented by antigenpresenting cells. The $\mathrm{T}$ cell antigen receptor is a membrane-bound molecule that recognizes peptide fragments of pathogens exposed in MHC molecules in antigen-presenting cells. B-lymphocyte has immunoglobulin molecules (Ig) on its surface, which is its antigen receptor. Antibodies are soluble forms of Igs secreted following activation of $\mathrm{B}$ cells and bind to pathogens and external material in extracellular space (humoral immunity) (Hajishengallis, 2015).

After antigenic stimulation, naïve CD4 $+\mathrm{T}$ cells are activated, proliferate and differentiate into subpopulations of distinct effector cells characterized by their specific cytokine production profiles and specific functions (Hernández et al., 2011). They may differentiate into several subpopulations, among then, Th1 and Th2. Increased T-bet expression, the transcription factors controlling Th1 differentiation, has been detected in active periodontal lesions and its over-expression correlated positively with enhanced expression of IL-1 $\beta$ and interferon- $\gamma$ (Hernández et al, 2011).

Th1 cells secrete IL- 2 , IFN- $\gamma$ and TNF- $\beta$ and are involved in control of intracellular pathogens and inflammatory diseases. Whereas Th2 cells produce IL-4, IL-5, IL-6, IL-10 and IL-13 (Linden et al., 2013).

Th1 cytokines (IL-2 and IFN- $\gamma$ ) boost cell-mediated responses, whereas Th2 cytokines (IL-4) suppress cellmediated responses. $\mathrm{T}$ CD4+ lymphocytes subpopulations also regulate $\mathrm{B}$ cells functions, directly affecting immunoglobulin class switching by B cells (Hajishengallis, 2015). Autoimmune reactions are evident in periodontal lesions through the production of autoantibodies against periodontal tissue components (collagen, desmosomal proteins, neutrophils, phospholipids) 
(Berglundh et al., 2007; Houri-Haddad et al., 2007; Garlet, 2010; Hajishengallis, 2014).

It is known that $\mathrm{Th}$ cell responses provide the necessary cytokines for proliferation of B cells and their polyclonal activation, facilitated by the presence of LPS, leading to high levels of antibodies and continuous production of IL-1 $\beta$, which contributes to bone resorption (Amunulla et al., 2008; Gaffen and Hajishengallis, 2008).

The discovery of the Th17 subpopulation propitiated to re-examine the role of $\mathrm{T}$ cells in inflammatory diseases (Hajishengallis, 2014). Two well-defined subpopulations of CD4+ T cells, Th17 and Tregs, when activated, perform antagonistic roles, as effector and suppressor cells, respectively and both are important in host response and pathogenesis of PD (Linden et al., 2013; Hajishengallis, 2014).

Although Tregs and Th17 cells perform different roles in the pathogenesis of infection, it has been shown that they have a common path of development. Naïve $T$ cells exposed to TGF- $\beta$ upregulate transcription factor Foxp3 and become induced Treg, which suppress inflammation. But when naïve cells are cultured with TGF- $\beta$ and IL-6, for instance, as occur during early phase of periodontitis, Th17 cells are generated and present pathological activities that are pro-inflammatory and pro-resorptive (Hernández et al., 2011).

Recently, an anti-inflammatory protective role mediated by IL-9 and IL-22, produced by Th9 and Th22 lymphocytes, respectively, was suggested in stable periapical lesions. IL-9 exerts proinflammatory or anti-inflammatory activities through the modulation of the development and function of Tregs and/or Th17. IL-22 is also characterized as a pleiotropic cytokine (Aranha et al., 2013).

Th17 cells act against extracellular bacteria and fungi that were phagocytosed by antigen presenting cells (APCs). Th17 cells also produce IL-6, IL-1 $\beta$, TNF- $\alpha$, IL-21, IL-22, IL-23, IL-26 and Receptor Activator of Nuclear factor Kappa B Ligand (RANKL) (Linden et al., 2013; Hajishengallis, 2014). The expansion of human Th17 cells is regulated by IL-23, whereas IL-1, TGF- $\beta 1$ and IL-6 initiate the differentiation of Th17 (Konermann et al., 2012). Moreover, IL-21, as an autocrine-regulating factor for Th17 cells, plays an important role in inducing the differentiation of Th17 cells and inhibition of Th1 and Treg function (Zhao et al., 2011).

Another critical aspect in PD is the control of bone homeostasis that depends on a dynamic balance between bone-forming osteoblasts and bone-resorbing osteoclasts that is tightly controlled by several regulatory mechanisms, such as endocrine system and immune response (Hernández et al., 2011). Osteoclasts induce periodontal bone resorption and the balance between bone resorption by osteoclasts and bone formation by osteoblasts controls the level of bone mass (Chen et al., 2014). The functional characterization of three new members of the TNF superfamily (ligand and receptor) has significantly contributed to the establishment of osteoimmunology and their participation as key modulators of physiology and pathology of bone resorption: RANKL, its receptor RANK and the soluble decoy receptor of RANKL, Osteoprotegerin (OPG) (Srinivasan, 2013). RANKL is a polypeptide of 314 amino acids expressed as membrane-bound protein or in secreted form and the OPG inhibits RANK/RANKL interaction (Chen et al., 2014). RANKL exerts its biological effects by inducing osteoclast differentiation, maturation, activation and inhibition of apoptosis (Hernández et al., 2011). In presence of Macrophage Colony Stimulating Factor (M-CSF), RANKL binds to RANK present in osteoclasts and Osteoclast Precursors (OCP) and activate these cells. In addition, many well-known osteotropic factors, including TNF- $\alpha$, PGE $_{2}$, IL-1 $\beta$, IL-6 and IL-17, exert their osteoclastogenic activity through the induction of RANKL expression in osteoblasts and CD4+ T cells (Hernández et al., 2011; Hienz et al., 2015). The level of RANKL mRNA and the RANKL/OPG mRNA ratio were enhanced in chronic periodontitis, was related to the number of $P$. gingivalis (Tuter et al., 2010; Vernal et al., 2004; Wara-Aswapati et al., 2007) and by inhibiting the RANKL/RANK signal there was a decrease in bone resorption during experimental periodontitis in rats (Jin et al., 2007).

A cytokine known as TRANCE (cytokine related to TNF), previously identified in CD4+ T cells, may induce differentiation and osteoclast activation and act directly in OCP and mature osteoclasts, through RANKL synthesis during diseases involving bone resorption (Hernández et al., 2011).

Transcription factor NF-kB binds to DNA sequences called $\mathrm{kB}$ sites, is essential for osteoclast formation and survival and regulates bone homeostasis by inducing the cytokines RANKL, TNF- $\alpha$ and IL-1 involved in periodontitis (Xu et al., 2009. Porphyromonas gingivalis and Fusobacterium nucleatum activate NF- $\kappa \mathrm{B}$ transcription in epithelial cells and upregulate proinflammatory cytokine gene transcripts (Abu-Amer, 2013). Bacterial lipopolysaccharides activate $\mathrm{NF}-\kappa \mathrm{B}$, osteoclastogenesis and osteolysis in mice (Abu-Amer et al., 1997).

Osteoblasts, $\mathrm{T}$ and $\mathrm{B}$ cells and fibroblasts express RANKL. Osteoblasts express TLR 1, 2, 4 and 6 and respond to TLR 2/6 and TLR 2/1 ligands, increasing the transcription of NF- $\kappa \mathrm{B}$ and RANKL expression. OPG, the bone protection factor, produced by osteoblasts and bone marrow stromal cells (fibroblasts residing in the periodontum and endothelial cells), inhibits RANK/RANKL interaction and prevents bone 
resorption (Kawai et al., 2006; Hans and Hans, 2011; Yokoyama et al., 2011).

Additionally, chemotactic Macrophage Inflammatory Protein (MIP)-3 $\alpha$ is crucial for the chemotaxis and maintenance of immune cells, especially Th17 cells in inflamed tissues. It has been suggested a possible role for periodontal ligament fibroblasts by upregulating the chemotactic MIP-3 $\alpha$ that attract Th17 cells into the inflamed periodontal tissues (Konermann et al., 2012), where these lymphocytes mediate tissue damage. Th17 lymphocyte may provide protection because they attract neutrophils and induce the expression of antimicrobial factors. However, they may also contribute to pathogenesis of autoimmune inflammatory disease, by causing osteoclastogenesis (IL-17 and RANKL) (Konermann et al., 2012). Th17 cells are observed at sites with $\mathrm{CP}$ and related Th17 cytokines are produced in periodontal lesions (Linden et al., 2013; Hajishengallis, 2014).

Recent studies have claimed that the bacteria $P$. gingivalis is a host response handler rather than a potent inducer of inflammation, i.e., it has activity usually associated with another bacterium involved in inflammatory disease. Specifically, by subverting the innate immune response, including the interference in complement pathway and TLRs, $P$. gingivalis can impair host defenses in such a way that the growth and development of the entire microbial community is altered, thereby causing a destructive change in its homeostatic relationship with the host. Furthermore, it is suggested that $P$. gingivalis can modify the adaptive immune response. The interaction of $P$. gingivalis with dendritic cells induces a pattern of cytokines, which promotes the polarization to Th17 lymphocytes, rather than to the effective Th1 lineage, which promotes immune clearance of $P$. gingivalis. $P$. gingivalis also inhibits chemokine production by gingival epithelial cells, which recruit Th1 cells, as well as IFN- $\gamma$ production by $\mathrm{T}$ cells (Hajishengallis, 2014).

IL-17 acts on cells of the innate and adaptive immune system and connective tissue cells, such as neutrophils, fibroblasts and osteoblasts. Through these interactions, IL-17 induces the production of CXC chemokines (cysteine-amino acid-cysteine, chemokine family, in which the cysteine residues are separated by one amino acid) that recruit neutrophils, Matrix Metalloproteinases (MMPs) and other molecules related to tissue destruction (Hajishengallis, 2014).

Thus, activated lymphocytes (B and $\mathrm{T}$ cells, specifically Th1 and Th17) play an important role in pathological bone resorption by the same mechanism dependent on RANKL (Hajishengallis, 2014). In addition, activated neutrophils express membrane-bound RANKL and can stimulate osteoclastogenesis directly if they are close enough to the bone. The anti-inflammatory cytokine IL-10 produced by Tregs, as well as IFN- $\gamma$ produced by Th1 cells and IL- 4 and IL-13 produced by Th2 cells can suppress osteoclastogenesis (Hajishengallis, 2014) (Fig. 1).

Recently, important hypotheses on the pathophysiological role of IL-33 in PD were reported. IL-33 is a recently discovered member of the IL-1 family. Endothelial and epithelial cells constitutively produce this cytokine. IL-33 is a ligand ST2 receptor, a member of the superfamily TLR/IL-1R. This cytokine probably plays three roles in relation to PD: As an alarm, a chemoattractant and a systemic cytokine. The release of IL-33 when operating as an alarm, results in the destruction of many cells, primarily fibroblasts and epithelial cells, by necrosis. In the context of inflammatory disease, IL-33 induces other responses such as degranulation of mast cells and production of pro-inflammatory cells (e.g., macrophages, eosinophils and basophils). The release of inflammatory mediators and IL-33 will induce osteoblast activation, which leads to the production of RANKL and diminishes OPG production and subsequent activation of osteoclasts. Moreover, mast cell degranulation, in addition to the inflammatory state, will induce sensitization of circulating monocytes, which will differentiate into osteoclasts in this microenvironment (da Luz et al., 2014).

The role of B cell and plasma cell is not fully understood in periodontitis. It is considered that the antibody response is not protective. In fact, increased deposition of immune complexes together with complement fragments in the diseased gum suggest that the antibodies secreted from plasma cells may be involved in the inflammatory response. The ability of B cells to produce inflammatory cytokines and MMPs can further contribute to tissue damage. Perhaps most importantly, B cells constitute, together with T cells, the biggest source of RANKL secreted and bound to the membrane in bone resorption lesions in periodontitis. The postulated protective role of Th1 cells is consistent with the negative correlation of Th1 cytokines (IFN $\gamma$ and IL-12) and the severity of periodontitis observed in some studies, the ability of these cytokines to promote immunity mediated by cells and to inhibit osteoclastogenesis. However, other studies have attributed the destructive effects to Th1 cells and IFN $\gamma$ in periodontitis, because the capacity of activated Th1 cells also to express RANKL. Such discrepancies can therefore be partly attributed to the opposing roles of the same subpopulation of $\mathrm{T}$ cells in periodontitis. Likewise, Th2 cells that promote destructive responses of B cells can also secrete IL-4 and IL-13, which can inhibit osteoclastogenesis (Hajishengallis, 2014). 


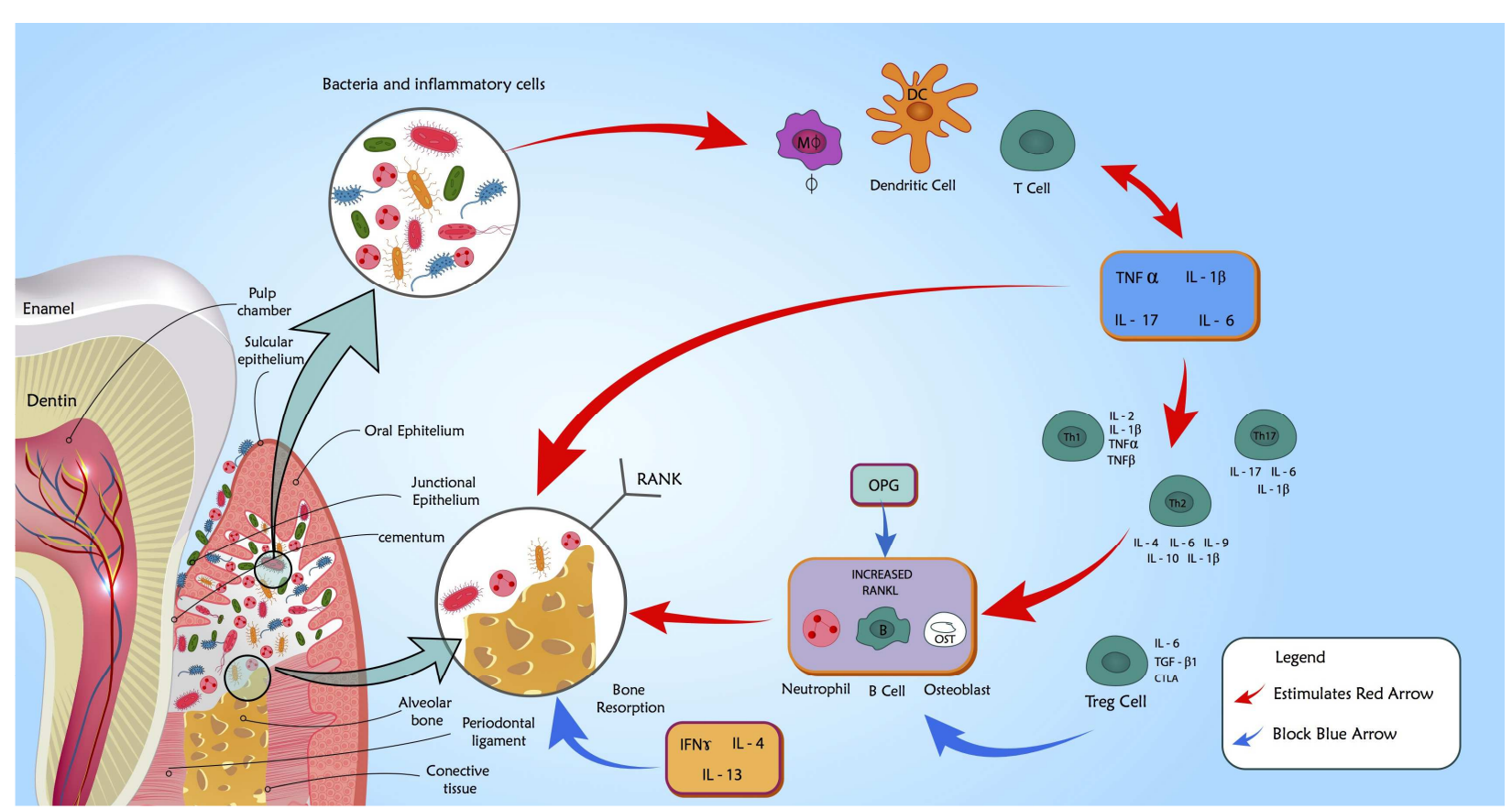

Fig. 1. This figure shows the anatomy of the periodontium around the tooth in periodontal disease. The epithelial barrier breached causes bacterial invasion of the underlying connective tissue, in which the resident immune cells in periodontium, such as macrophages, dendritic cells, $\mathrm{T}$ cells and $\mathrm{B}$ cells, are activated. This situation favors the exaggerated release of proinflammatory cytokines (TNF- $\alpha$, IL-1 $\beta$, IL-6, IL-17, etc.), which lead to the differentiation and activation of $T$ cell subpopulations. Activated Th1, Th2 and Th17 release specific cytokines of each polarized T cell subpopulation and thus promote the activation of Receptor Activator of Nuclear Factor Kappa beta Ligand (RANKL) expression on the membranes of neutrophils, B cells, periodontal ligament fibroblasts and osteoblasts, which induces bone resorption in periodontal disease after binding to its RANK receptor on the osteoclast membrane. The proliferation and activation of Treg cells promote the release of cytokines, which have anti-inflammatory roles in osteoclastogenesis and thus prevents bone resorption in the active sites of the disease. Osteoprotegerin (OPG) has an inhibitory role in osteoclastogenesis

Thus, the dissection of the periodontal response of the host in terms of protective and destructive aspects seems to be confused by the nature of the disease, in which a powerful antimicrobial protective response can be compensated by collateral inflammatory tissue damage. This also possibly becomes true for other complex inflammatory diseases with complex polymicrobial etiology. Currently, therefore, it is not possible to attribute definitive functions for effector $\mathrm{T}$ cell subpopulations in periodontitis (Hajishengallis, 2014).

It is likely that different subpopulations of $\mathrm{T}$ cells predominate at different stages of PD and the inability to clinically determine the activity of the disease becomes an important limitation in all studies (Gemmell et al., 2002).

\section{Local and Systemic Responses in Periodontal Disease}

In crevicular cells (gingival fluid), significant differences were established between patients with localized AgP (LAgP), generalized AgP (GAgP), CP and controls in relation to the average number of cytotoxic $\mathrm{T}$ lymphocytes- CD8+ (LAgP > CP and controls) and Blymphocytes- $\mathrm{CD} 20+(\mathrm{LAgP} / \mathrm{GAgP}>\mathrm{CP}$ and
LAgP/GAgP > controls). Significant variations were observed in the rate of $\mathrm{T}$-helper lymphocytes CD4+ $/ \mathrm{CD} 8+(\mathrm{LAgP}<$ controls and $\mathrm{GAgP}<$ controls $)$ as well as in the correlation between the number of $\mathrm{T}$ cells and the degree of inflammation. This supports the hypothesis of altered immunoinflammatory pathogenesis in patients with AgP (Sigusch et al., 2006).

CD4+ and CD8+ cells predominate in samples of gingival biopsies from patients with $\mathrm{CP}$. In contrast, in patients with $\mathrm{AgP}$, all four-cell types (CD4+, $\mathrm{CD} 8+, \mathrm{CD} 20+$ (plasma cells) and CD68+ cells (macrophages)) were significantly increased. CD20+ cells were significantly more prevalent in AgP than in CP (Artese et al., 2011).

Serum levels of IFN- $\gamma$, TNF- $\alpha$ and IL-10 were significantly increased, while IL-2 was significantly lower in periodontitis patients compared with healthy controls. An increased serum level of IFN- $\gamma$ and TNF- $\alpha$ in patients with periodontitis was associated with an increase in A. actinomycetemcomitans and $P$. gingivalis in dental biofilm, respectively (Andrukhov et al., 2011).

In addition, the humoral immune response to bacteria is stimulated in PD patients, as determined by serum IgG 
antibodies to specific bacteria and/or their antigens (Ebersole et al., 1987).

Although some studies have shown that severe forms of PD have similar systemic inflammatory profiles (Cairo et al., 2010; D'Aiuto et al., 2010; Picolos et al., 2005; Lima et al., 2011) found different cell sources of immunoregulatory cytokines in patients with AgP compared to patients with $\mathrm{CP}$, in which the frequency of IL-10 in cells that express CD14+ was higher in CP but not in AgP in comparison to healthy controls. The amount of CD4+ T cells involved in IL-4 production was higher in $\mathrm{CP}$ than in healthy controls.

Evidence that periodontitis constitutes a disease in which an infectious and inflammatory factor is capable of increasing the synthesis of inflammatory mediators is shown by the relevant contribution that studies involving periodontal therapy have provided. Nonsurgical periodontal therapy reduced the levels of IL-17 and IL21 and increased the levels of IL-4 in gingival fluid; however, there were no significant differences in the levels of IFN- $\gamma$. Furthermore, the amount of Th17 cells in peripheral blood was reduced, especially the IL-17+ and IFN $-\gamma+$ subpopulations. These results suggested that Th17 cells play a destructive role in the immune balance in periodontitis, the effect of Th1 cells was not significant, while Th2 cells had a protective effect (Zhao et al., 2011). In addition, it is known that individuals with better response to periodontal therapy decreased their inflammatory risk category (OR 4.8, 95\% CI 1.4 to 15.8 ) after correction for age, gender, ethnicity and tobacco use (D'Aiuto et al., 2004a).

Note that the effectiveness clearly established by periodontal therapy in the resolution of inflammation and healing of periodontal tissues involves changing in the microbiota, with recolonization by commensal bacteria and restoration of homeostasis. This yields, among other benefits, reduction in production of inflammatory mediators and consequently a decrease or absence of their systemic effects.

\section{Discussion}

In individuals susceptible to periodontitis, absence of resolution of periodontal inflammation results in chronic inflammation, which may have a systemic impact (D'Aiuto et al., 2013; Dietrich et al., 2013; Ide and Papapanou, 2013; Linden and Herzberg, 2013; Linden et al., 2013; Taylor et al., 2013). The acute inflammatory response is protective, but failure to remove the inflammatory cells, especially neutrophils, promotes chronic, pathological and destructive lesions. Therefore, it is evident that the three aspects of the pathogenesis of periodontitis (infection, inflammation and adaptive immunity) have a potential role and impact on the systemic immunoinflammatory response, which either initiates or mediates a wide range of systemic diseases (Linden et al., 2013).

In a previous study by our group, we found levels of high-sensitivity C-reactive protein (hs-CRP) $>0.3$ $\mathrm{mg} / \mathrm{dL}$ in individuals with severe periodontitis compared to controls (60.87 versus 23.08, respectively; $p=0.0216$ ) (Leite et al., 2014) and periodontal therapy was associated with a decrease in hs-CRP levels circulating in serum and an increase in High Density Lipoprotein (HDL).

Moreover, WBC count is characterized as a crude marker of systemic inflammation and correlates to the host response with respect to a variety of stimuli. This marker has also been associated with a significant prediction of future cardiovascular events and glucose intolerance in different populations (D'Aiuto et al., 2006; Graziani et al., 2010).

Earlier studies (do Vale et al., 2004; Figueira et al., 2009) showed that peripheral blood mononuclear cells from patients with periodontitis did not proliferate in response to bacterial antigens. However, other studies (Pejcic et al., 2011; Gaddale et al., 2014) have confirmed an increased number of WBCs and neutrophils in the peripheral blood of patients with moderate to severe periodontitis, with a positive correlation between disease severity and number of WBCs in the blood.

Additionally, rigorous therapeutic protocols and periodontal maintenance contributed to the restoration of phagocytic function in peripheral blood neutrophils (Carneiro et al., 2012) and a reduction in hs-CRP levels in patients with severe periodontitis (Leite et al., 2014). These findings also support the conclusions of D'Aiuto et al. (2004b; 2004c) and Kamil et al. (2011) when reporting a greater reduction in the levels of systemic inflammatory markers among those with better clinical responses to periodontal therapy.

Conversely, serum levels of various cytokines in patients with periodontitis were investigated in several previous studies and the results were often controversial (Duarte et al., 2010; Andrukhov et al., 2011; Zhao et al., 2011). The contradictions in the existing data are probably caused by the heterogeneity among the various individuals, differences in susceptibility to periodontitis, differences in the oral microbiota and low levels of cytokines in the serum (Andrukhov et al., 2011).

Since Th1, Th2 and Th17 are mutually inhibitory, it can be argued that the simultaneous action of $\mathrm{T}$ cells in the development of periodontitis is unlikely and that these subpopulations of $\mathrm{T}$ cells can lead to disease progression independently. However, more studies are necessary for the accurate determination of crosstalk between $\mathrm{T}$ helper cytokines in periodontitis and its impact on the evolution of the disease (Garlet, 2010).

Different combinations of adhesion molecules and their ligands, or the expression of different cytokines and 
chemokines in the microenvironment, contribute for differences in the recirculation or selective recruitment of lymphocyte subsets to sites of inflammation, which in turn may influence the outcome of disease (Seymour et al., 1993; Lima et al., 2011).

The unique feature of the biofilm in the oral cavity, particularly the subgingival biofilm, is its close proximity to highly vascularized tissues. Any disruption of natural integrity of the subgingival epithelium, whose thickness is at most 10 layers, can lead to bacteremia (Parahitiyawa et al., 2009). Furthermore, in periodontitis, the periodontal pocket epithelium is typically thin and ulcerated and, therefore, often opens, allowing access of pathogens to the connective tissue and blood vessels. In patients with moderate to severe periodontitis, the total area of the pocket epithelium in direct contact with the subgingival biofilm is surprisingly large, reaching to about the size of the palm of the human hand or much larger in advanced cases (Page, 1998). Therefore, both the access of microorganisms to the bloodstream and the onset of chronic inflammation with area and intensity sufficient to elicit a significant host response provide the basis for the study of the inter-relationship between periodontitis and CVD and obesity, among others.

Thus, nonsurgical periodontal therapy acts to reduce inflammation markers in the blood CRP, IL-6, etc.) and reveals some important aspects of the systemic reach of periodontal inflammation (Fitzsimmons et al., 2010; Zhao et al., 2011). However, it is emphasized that the mechanical removal of the dental biofilm by scaling and root planning, with or without another supporting modality, does not mean that, at any magnitude, inflammation/infection does not persist. Sometimes the removal of local factors is only achieved after repeated specific actuations principally at sites in which there still exist clinical signs of inflammation. Sites with residual periodontal pockets that continue bleeding on probing undoubtedly require new instrumentation (scaling and root planning) and reinforcement of oral hygiene instruction. Moreover, the need for reinstrumentation and clinical response are unique to each individual patient and for this reason, there is no way to generically establish the completion of therapy for all patients at the same time.

New treatments for periodontitis should address the important contribution of immune cells in bone resorption, particularly with regard to the natural course of the disease (remission and progression periods) (Hajishengallis, 2014). Current treatments for periodontitis, despite of obtaining excellent local and systemic clinical results, depend on mechanical procedures (scaling and root planning), focusing mainly in the dental biofilm that should be destroyed and eliminated whenever possible, however it neglects, in the most part, the immunological cells. However, based on the current understanding of immune response, it is essential to re-evaluate the forms of treatment currently available, adding possible new therapeutic strategies to control alveolar bone loss caused by the cell-mediated immune response. As a possible target, inhibit the production and/or expression of the RANKL (by acting in transcription and/or translation) by activated immune cells and/or block the RANKL-RANK physiological interaction may be suggested. Although animal models have shown promising results for several therapeutic approaches, problems of side effects also arise.

It is also known that the absence of Tregs is an indication of a great variety of disorders, such as autoimmunity, dermatitis, periodontitis and even transplant rejection. A potential treatment option for these disorders revolves around increasing the number of Tregs in local sites (Jhunjhunwala et al., 2012; Gonzales et al., 2013). Current methods for in vivo expansion of Tregs depend on biological therapies, which are not specific for Tregs and are still associated with many adverse side effects. Synthetic formulations capable of inducing Tregs could be an alternative strategy to achieve an in situ increase in the number of Tregs. The in vitro tests of a synthetic formulation of Treg inducers that consist of controlled release vehicles of IL-2, TGF- $\beta$ and rapamycin were already reported, using cytokine and/or drug combination. IL-2, TGF- $\beta$ and rapamycin are released over three to four weeks from these formulations. Furthermore, Tregs induced by these formulations expressed the established markers for Tregs (phenotype) and suppressed the proliferation and function of naïve $T$ cells to levels similar to that of the natural soluble factor of Tregs, as well as naturally occurring Tregs lymphocytes. These formulations were also able to induce transcription factors FoxP3+ Tregs in human cells in vitro. The authors concluded that the combination of these microparticle formulations have the potential to be used for the in vivo induction of Tregs at local sites of rejected transplants or autoimmunity (Jhunjhunwala et al., 2012).

Periodontitis is characterized by polymicrobial infection, so that, pathogens can modulate the $\mathrm{T}$ cell response to promote its own adaptability and the immune response itself becomes a mixture of immunological responses mediated by all the microorganisms represented in the biofilm (Garlet, 2010; Hajishengallis, 2014). Therefore, it may not be possible to reliably dissect patterns of dominant activities of Th1, Th2, Th17 or Tregs among the diseased periodontal tissue samples collected. Thus, it may be simpler and more productive to consider the roles played by each cytokine (Th1, Th2, Th17 or Tregs) in periodontal infection, since the performance of $\mathrm{T}$ cells in the maintenance of homeostasis between the biofilm microorganisms and host is remarkable. 
It is unclear which specific signaling pathways need to be blocked or enhanced to mitigate the disease in order to promote host defense. Moreover, the modulation of the host immune system by drugs may result in adverse side effects, requiring close monitoring of patients for trying this approach (Jhunjhunwala et al., 2012). Therefore, additional studies are needed to understand the onset and progression of periodontitis and to develop therapeutic interventions able to treat and control the disease.

\section{Conclusion}

PD leads to an array of events involving the innate and adaptive immunity in affected host, which in turn brings the need for a growing understanding of the immunopathogenesis of periodontitis in order to improve the treatment and control of the disease.

New therapeutic approaches that include the current understanding of the genetic factors and the host (genome, proteomic analysis, transcription factors, cytokines, immune cells) as well as the complex microbial community (metagenomic, metatranscriptomic) should be tested in animal models and epidemiological studies and extended to clinical trials for the control of the overall risk of periodontitis and associated systemic diseases.

\section{Acknowledgement}

The authors gratefully acknowledge Mr. Derrick Mulder for reviewing the English language of the manuscript. Maria Imaculada Muniz-Junqueira is an investigator supported by the Conselho Nacional de Desenvolvimento Científico e Tecnológico (CNPq), Brazil (process number 308985/2013-3).

\section{Author's Contributions}

All the authors contributed to the writing and revision of the manuscript.

\section{Ethics}

This article is original and contains unpublished material. The corresponding author confirms that all of the other authors have read and approved the manuscript and no ethical issues involved.

\section{References}

AAP, 1999. Consensus report: Chronic periodontitis. 1999 Ann. Periodontol., 4: 38-38. DOI: 10.1902/annals.1999.4.1.38

Aas, J.A., B.J. Paster, L.N. Stokes, I. Olsen and F.E. Dewhirst, 2005. Defining the normal bacterial flora of the oral cavity. J. Clin. Periodontol., 43: 57215732. DOI: $10.1128 /$ JCM.43.11.5721-5732.2005
Abu-Amer, Y., F.P. Ross, J. Edwards and S.L. Teitelbaum, 1997. Lipopolysaccharide-stimulated osteoclastogenesis is mediated by tumor necrosis factor via its P55 receptor. J. Clin. Investigat., 100: 1557-1565. DOI: 10.1172/JCI119679

Abu-Amer, Y., 2013. NF- $\mathrm{B}$ signaling and bone resorption. Osteoporosis Int., 24: 2377-2386. DOI: $10.1007 / \mathrm{s} 00198-013-2313-\mathrm{x}$

Amunulla, A., R. Venkatesan, H. Ramakrishnan, K.V. Arun and S. Sudarsan et al., 2008. Lymphocyte subpopulation in healthy and diseased gingival tissue. J. Ind. Society Periodontol., 12: 45-50. DOI: $10.4103 / 0972-124 X .44091$

Andrukhov, O., C. Ulm, H. Reischl, P.Q. Nguyen and M. Matejka et al., 2011. Serum cytokine levels in periodontitis patients in relation to the bacterial load. J. Periodontol., 82: 885-892. DOI: $10.1902 /$ jop.2010.100425

Aranha, A.M.F., C.E. Repeke, T.P. Garlet, A.E. Vieira and A.P. Campanelli et al., 2013. Evidence supporting a protective role for Th9 and Th22 cytokines in human and experimental periapical lesions. J. Endodont., 39: 83-87. DOI: $10.1016 /$ j.joen.2012.10.015

Armitage, G.C., 1999. Development of a classification system for periodontal diseases and conditions. Annals Periodontol., 4: 1-6. DOI: 10.1902/annals.1999.4.1.1

Armitage, G.C. and M.P. Cullinan, 2010. Comparison of the clinical features of chronic and aggressive periodontitis. Periodontology, 53: 12-27. DOI: $10.1111 /$ j.1600-0757.2010.00353.x

Artese, L., M.J. Simon, A. Piattelli, D.S. Ferrari and L.A.G. Cardoso et al., 2011. Immunohistochemical analysis of inflammatory infiltrate in aggressive and chronic periodontitis: A comparative study. Clin. Oral Investigat., 15: 233-240. DOI: $10.1007 / \mathrm{s} 00784-009-0374-1$

Berglundh, T., M. Donati and N. Zitzmann, 2007. B cells in periodontitis-friends or enemies? Periodontology, 45: 51-66. DOI: 10.1111/j.1600-0757.2007.00223.x

Cairo, F., M. Nieri, A.M. Gori, P. Tonelli and R. Branchi et al., 2010. Markers of systemic inflammation in periodontal patients: Chonic versus aggressive periodontitis. An explorative crosss-sectional study. Eur. J. Oral Implantol., 3: 147-153. PMID: 20623039

Carneiro, V.M., A.C. Bezerra, M. Guimarães and M.I. Muniz-Junqueira, 2012. Effects of periodontal therapy on phagocytic activity of peripheral blood neutrophils-evidence for an extrinsic cellular defect. Oral Health Preventive Dentistry, 10: 195-203. DOI: $10.3290 /$ j.ohpd.a28008

Chen, B., W. Wu, W. Sun, Q. Zhang and F. Yan et al., 2014. RANKL expression in periodontal disease: Where does RANKL come from? BioMed Res. Int., 2014: 731039-731039. DOI: 10.1155/2014/731039 
D’Aiuto, F., L. Nibali, V. Mohamed-Ali, P. Vallance and M.S. Tonetti, 2004a. Periodontal therapy: A novel non-drug-induced experimental model to study human inflammation. J. Periodontal Res., 39: 294-299. DOI: 10.1111/j.1600-0765.2004.00741.x

D'Aiuto, F., M. Parkar, G. Andreaou, P.M. Brett and D. Ready et al., 2004b. Periodontitis and atherogenesis: Causal association or simple coincidence? A pilot intervention study. J. Clin. Periodontol., 31: 402-411. DOI: 10.1111/j.1600-051X.2004.00580.x

D'Aiuto, F., M. Parkar, G. Andreou, J. Suvan and P.M. Brett et al., 2004c. Periodontitis and systemic inflammation: Control of the local infection is associated with a reduction in serum inflammatory markers. J. Dental Res., 83: 156-160. DOI: $10.1177 / 154405910408300214$

D'Aiuto, F., M. Parkar, P.M. Brett, D. Ready and M.S. Tonetti, 2004d. Gene polymorphisms in proinflammatory cytokines are associated with systemic inflammation in patients with severe periodontal infections. Cytokine, 28: 29-34. DOI: $10.1016 /$ j.cyto.2004.06.005

D'Aiuto, F., M. Parkar, L. Nibali, J. Suvan and J. Lessem et al., 2006. Periodontal infections cause changes in traditional and novel cardiovascular risk factors: Results from a randomized controlled clinical trial. Am. Heart J., 151: 977-984.

DOI: $10.1016 /$ j.ahj.2005.06.018

D'Aiuto, F., L. Nibali, M. Parkar, K. Patel and J. Suvan et al., 2010. Oxidative stress, systemic inflammation and severe periodontitis. J. Dental Res., 89: 1241-1246. DOI: $10.1177 / 0022034510375830$

D'Aiuto, F., M. Orlandi and J.C. Gunsolley, 2013. Evidence that periodontal treatment improves biomarkers and CVD outcomes. J. Clin. Periodontol., 40: S85-S105.

DOI: 10.1902/jop.2013.134007

de Brito Bezerra, B.B., O. Andriankaja, J. Kang, S. Pacios and H.J. Bae et al., 2012. A. actinomycetemcomitans induced periodontal disease promotes systemic and local responses in rat periodontium. J. Clin. Periodontol., 39: 333-341. DOI: 10.1111/j.1600-051X.2011.01847.x

da Luz, F.A., A.P. Oliveira, D. Borges, P.C. Brígido and M.J. Silva, 2014. The physiopathological role of IL33: New highlights in bone biology and a proposed role in periodontal disease. Mediators Inflammat., 2014: 342410-342410. DOI: 10.1155/2014/342410

Dietrich, T., P. Sharma, C. Walter, P. Weston and J. Beck, 2013. The epidemiological evidence behind the association between periodontitis and incident atherosclerotic cardiovascular disease. J. Clin. Periodontol., 40: S70-S84. DOI: 10.1111/jepe.12062
Divaris, K., K.L. Monda, K.E. North, A.F. Olshan and L.M. Reynolds et al., 2013. Exploring the genetic basis of chronic periodontitis: A genome-wide association study. Human Molecular Genet., 22: 2312-2324. DOI: $10.1093 / \mathrm{hmg} / \mathrm{ddt} 065$

do Vale, C.H., L.A. de Oliveira Fraga, A.S. Costa, C.A. Tavares and O.A. Martins-Filho et al., 2004. Antiproliferative activity of Actinobacillus (Haemophilus) actinomycetemcomitans and Fusobacterium nucleatum in peripheral blood mononuclear cells. Res. Microbiol., 155: 731-740. DOI: 10.1016/j.resmic.2004.05.008

Duarte, P.M., M. da Rocha, E. Sampaio, M.J. Mestnik and M. Feres et al., 2010. Serum levels of cytokines in subjects with generalized chronic and agressive periodontitis before and after non-surgical periodontal therapy: A pilot study. J. Periodontol., 81: 1056-1063. DOI: 10.1902/jop.2010.090732

Dye, B.A., K. Choudhary, S. Shea and P.N. Papapanou, 2005. Serum antibodies to periodontal pathogens and markers of systemic inflammation. J. Clin. Periodontol., 32: 1189-1199. DOI: $10.1111 / \mathrm{j} .1600-051 X .2005 .00856 . x$

Ebersole, J.L., M.A. Taubman, D.J. Smith, D.E. Frey and A.D. Haffajee et al., 1987. Human serum antibody responses to oral microorganisms. IV. Correlation with homologous infection. Oral Microbiol. Immunol., 2: 53-59. DOI: 10.1111/j.1399-302X.1987.tb00290.x

Figueira, E.A., M.L.R. de Rezende, S.A.Torres, G.P. Garlet and V.S. Lara et al., 2009. Inhibitory signals mediated by programmed death-1 are involved with T-cell function in chronic periodontitis. J. Periodontol., 80: 1833-1844. DOI: 10.1902/jop.2009.090057

Fitzsimmons, T.R., A.E. Sanders, P.M. Bartold and G.D. Slade, 2010. Local and systemic biomarkers in gingival crevicular fluid increase odds of periodontitis. J. Clin. Periodontol., 37: 30-36. DOI: 10.1111/j.1600-051X.2009.01506.X

Gaddale, R., J.A. Mudda, I. Karthikeyan, S.R. Desai and H. Shinde et al., 2014. Changes in cellular and molecular components of peripheral blood in patients with generalized aggressive periodontitis. J. Investigative Clin. Dentistry. DOI: $10.1111 /$ jicd.12127

Gaffen, S.L. and G. Hajishengallis, 2008. A new inflammatory cytokine on the block: Re-thinking periodontal disease and the Th1/Th2 paradigm in the context of Th17 cells and Il-17. J. Dental Res., 87: 817-828. DOI: $10.1177 / 154405910808700908$

Garlet, G.P., 2010. Destructive and protective roles of cytokines in periodontitis: A re-appraisal from host defense and tissue destruction viewpoints. J. Dental Res., 89: 1349-1363. DOI: $10.1177 / 0022034510376402$

Gemmell, E. and G.J. Seymour, 1998. Cytokine profiles of cells extracted from humans with periodontal diseases. J. Dental Res., 77: 16-26. DOI: $10.1177 / 00220345980770010101$ 
Gemmell, E., C.L. Carter and G.J. Seymour, 2001. Chemokines in human periodontal disease tissues. Clin. Exp. Immunol., 125: 134-141. DOI: 10.1046/j.1365-2249.2001.01511.x

Gemmell, E., K. Yamazaki and G.J. Seymour, 2002. Destructive periodontitis lesions are determined by the nature of the lymphocytic response. Critical Rev. Oral Biol. Med., 13: 17-34. DOI: $10.1177 / 154411130201300104$

Glowacki, A.J., S. Yoshizawa, S. Jhunjhunwala, A.E. Vieira and G.P. Garlet et al., 2013. Prevention of inflammation-mediated bone loss in murine and canine periodontal disease via recruitment of regulatory lymphocytes. Proc. Nat. Acad. Sci. USA., 110: 18525-18530. DOI: 10.1073/pnas.1302829110

Gonzales, J.R., S. Groeger, A. Johansson and J. Meyle, 2013. T helper cells from aggressive periodontitis patients produce higher levels of interleukin-1 beta and interleukin-6 in interaction with Porphyromonas gingivalis. Clin. Oral Investig., 18: 1835-43.

DOI: $10.1007 / \mathrm{s} 00784-013-1162-5$

Gonzalez, O.A., M.J. Novak, S. Kirakodu, L. Orraca and K.C. Chen et al., 2014. Comparative analysis of gingival tissue antigen presentation pathways in ageing and periodontitis. J. Clin. Periodontol., 41: 327-339. DOI: $10.1111 /$ jepe. 12212

Graziani, F., S. Cei, M. Tonetti, M. Paolantonio and R. Serio et al., 2010. Systemic inflammation following non-surgical and surgical periodontal therapy. J. Clin. Periodontol., 37: 848-854. DOI: $10.1111 / \mathrm{j} .1600-051 \mathrm{X} .2010 .01585 . \mathrm{X}$

Guerrini, M.M. and H. Takayanagi, 2014. The immune system, bone and RANKL. Archives Biochem. Biophys., 561: 118-123. DOI: $10.1016 /$ j.abb.2014.06.003

Hajishengallis, G., 2014. Immunomicrobial pathogenesis of periodontitis: Keystones, pathobionts and host response. Trends Immunol., 35: 3-11.

DOI: 10.1016/j.it.2013.09.001

Hajishengallis, G., 2015. Periodontitis: From microbial immune subversion to systemic inflammation. Nature Rev. Immunol., 15: 30-44.

DOI: $10.1038 /$ nri3785.

Hanada, R., T. Hanada, V. Sigl, D. Schramek and J.M. Penninger, 2011. RANKL/RANK-beyond bones. J. Molecular Med., 89: 647-656.

DOI: $10.1007 / \mathrm{s} 00109-011-0749-\mathrm{z}$

Hans, M. and V.M. Hans, 2011. Toll-like receptors and their dual role in periodontitis: A review. J. Oral Sci., 53: 263-271. DOI: 10.2334/josnusd.53.263

Hernández, M., N. Dutzan, J. García-Sesnich, L. Abusleme and A. Dezerega et al., 2011. Hostpathogen interactions in progressive chronic periodontitis. J. Dental Res., 90: 1164-1170. DOI: $10.1177 / 0022034511401405$
Hienz, S.A., S. Paliwal and S. Ivanovski, 2015. Mechanisms of bone resorption in periodontitis. J. Immunol. Res., 2015: 615486-615495. DOI: $10.1155 / 2015 / 615486$

Houri-Haddad, Y., A. Wilensky and L. Shapira, 2007. Tcell phenotype as a risk factor for periodontal disease. Periodontology, 45: 67-75. DOI: 10.1111/j.1600-0757.2007.00227.x

Ide, M. and P.N. Papapanou, 2013. Epidemiology of association between maternal periodontal disease and adverse pregnancy outcomes-systematic review. J. Clin. Periodontol., 40: S181-S194.

DOI: $10.1902 /$ jop.2013.134009

Jhunjhunwala, S., S.C. Balmert, G. Raimondi, E. Dons and E.E. Nichols et al., 2012. Controlled release formulations of IL-2, TGF- $\beta 1$ and rapamycin for the induction of regulatory T cells. J. Controlled Release, 159: 78-84. DOI: $10.1016 /$ j.jconrel.2012.01.013

Jin, Q., J.A. Cirelli, C.H. Park, J.V. Sugai and M. Taba Jr. et al., 2007. RANKL inhibition through osteoprotegerin blocks bone loss in experimental periodontitis. J. Periodontol., 78: 1300-1308. DOI: 10.1902/jop.2007.070073

Kamil, W., R. Al Habashneh, Y. Khader, L. Al Bayati and D. Taani, 2011. Effects of nonsurgical periodontal therapy on C-reactive protein and serum lipids in Jordanian adults with advanced periodontitis. J. Periodontal Res., 46: 616-621. DOI: 10.1111/j.1600-0765.2011.01380.x

Kawai, T., T. Matsuyama, Y. Hosokawa, S. Makihira and M. Seki et al., 2006. B and T lymphocytes are the primary sources of RANKL in the bone resorptive lesion of periodontal disease. Am. J. Pathol., 169: 987-998. DOI: 10.2353/ajpath.2006.060180

Kobayashi, R., T. Kono, B.A. Bolerjack, Y. Fukuyama and R.S. Gilbert et al., 2011. Induction of IL-10producing CD4+ T-cells in chronic periodontitis. J. Dental Res., 90: 653-658. DOI: $10.1177 / 0022034510397838$

Konermann, A., M. Beyer, J. Deschner, J.P. Allam and N. Novak et al., 2012. Human periodontal ligament cells facilitate leukocyte recruitment and are influenced in their immunomodulatory function by Th17 cytokine release. Cellular Immunol., 272: 137-143. DOI: 10.1016/j.cellimm.2011.10.020

Leite, A.C.E., V.M.A. Carneiro and M.C.M. Guimarães, 2014. Effects of periodontal therapy on C-reactive protein and HDL in serum of subjects with periodontitis. Revista Brasileira de Cirurgia Cardiovascular, 29: 69-77. DOI: $10.5935 / 1678-9741.20140013$

Pejcic, A., L. Kesic, Z. Pesic D. Mirkovic and M. Stojanovic, 2011. White blood cell count in different stages of chronic periodontitis. Acta Clinica Croata, 50: 159-167. DOI: 10.5935/1678-9741.20140013 
Lima, P.M.A., P.E.A. Souza, J.E.Costa, R.S. Gomez and K.J. Gollob et al., 2011. Aggressive and chronic periodontitis correlate with distinct cellular sources of key immunoregulatory cytokines. J. Periodontol., 82: 86-95. DOI: 10.1902/jop.2010.100248

Linden, G.J. and M.C. Herzberg, 2013. Periodontitis and systemic diseases: A record of discussions of working group 4 of the Joint EFP/AAP Workshop on Periodontitis and Systemic Diseases. J. Clin. Periodontol., 40: S20-S23. DOI: 10.1111/jcpe.12091

Linden, G.J., A. Lyons and F.A. Scannapieco, 2013. Periodontal systemic associations: Review of the evidence. J. Clin. Periodontol., 40: S8-S19. DOI: $10.1111 /$ jcpe. 12064

Loo, W.T.Y., Y. Yue, C.B. Fan, L.J. Bai and Y.D. Dou et al., 2012. Comparing serum levels of cardiac biomarkers in cancer patients receiving chemotherapy and subjects with chronic periodontitis. J. Translat. Med., 10: 1-7. DOI: 10.1186/1479-5876-10-S1-S5

Maekawa, T., K. Tabeta, K. Kajita-Okui, T. Nakajima and K. Yamazaki, 2011. Increased expression of Creactive protein gene in inflamed gingival tissues could be derived from endothelial cells stimulated with interleukin-6. Archives Oral Biol., 56: 312-318. DOI: $10.1016 /$ j.archoralbio.2011.04.010.

Mombelli, A., F. Casagni and P.N. Madianos, 2002. Can presence or absence of periodontal pathogens distinguish between subjects with chronic and aggressive periodontitis? A systematic review. J. Clin. Periodontol., 29: 10-21. DOI: $10.1038 /$ sj.ebd.6400204

Moutsopoulos, N.M., J. Konkel, M. Sarmadi, M.A. Eskan and T. Wild et al., 2014. Defective neutrophil recruitment in leukocyte adhesion deficiency type I disease causes local IL-17-driven inflammatory bone loss. Sci. Translat. Med., 6: 229ra40-229ra40. DOI: 10.1126/scitranslmed.3007696.

Offenbacher, S., J.R. Elter, D. Lin and J.D. Beck, 2005. Evidence for periodontitis as a tertiary vascular infection. J. Int. Acad. Periodontol., 7: 39-48. PMID: 15912923

Ohlrich, E.J., M.P. Cullinan and G.J. Seymour, 2009. The immunopathogenesis of periodontal disease. Australian Dental J., 54: 2-10. DOI: 10.1111/j.1834-7819.2009.01139.x

Page, R.C., 1998. The pathobiology of periodontal diseases may affect systemic diseases: Inversion of a paradigm. Ann. Periodontol., 3: 108-120. DOI: 10.1902/annals.1998.3.1.108

Papapanou, P.N., 1999. Epidemiology of periodontal disease: An update. J. Int. Acad. Periodontol., 1: 110-116. PMID: 12666955

Papapanou, P.N., A.M. Neiderud, E. Disick, E. Lalla and G.C. Miller et al., 2004. Longitudinal stability of serum immunoglobulin $G$ responses to periodontal bacteria. J. Clin. Periodontol., 31: 985-990. DOI: 10.1111/j.1600-051X.2004.00599.x
Parahitiyawa, N.B., L.J. Jin, W.K. Leung, W.C. Yam and L.P. Samaranayake, 2009. Microbiology of odontogenic bacteremia: Beyond endocarditis. Clin. Microbiol. Rev., 22: 46-64. DOI: $10.1128 / C M R .00028-08$

Picolos, D.K., J. Lerche-Sehm, A. Abron, F.B Fine and P.N. Papapanou, 2005. Infection patterns in chronic and aggressive periodontitis. J. Clin. Periodontol., 32: $1055-1061$.

DOI: 10.1111/j.1600-051X.2005.00828.x

Sanz, M. and A.J.V. Winkelhoff, 2011. Periodontal infections: Understanding the complexityConsensus of the Seventh European Workshop on Periodontology. J. Clin. Periodontol., 38: 3-6. DOI: $10.1111 /$ j.1600-051X.2010.01681.x

Seymour, G.J., E. Gemmell, R.A. Reinhardt, J. Eastcott and M.A. Taubman, 1993. Immunopathogenesis of chronic inflammatory periodontal disease: Cellular and molecular mechanisms. J. Periodontal Res., 28: 478-486. DOI: 10.1111/j.1600-0765.1993.tb02108.x

Sigusch, B.W., A. Wutzler, T. Nietzsch and E. Glockmann, 2006. Evidence for a specific crevicular lymphocyte profile in aggressive periodontitis. J. Periodontal Res., 41: 391-396. DOI: 10.1111/j.1600-0765.2006.00869.x

Slade, G.D., S. Offenbacher, J.D. Beck, G. Heiss and J.S. Pankow, 2000. Acute-phase inflammatory response to periodontal disease in the US population. J. Dental Res., 79: 49-57. DOI: $10.1177 / 00220345000790010701$

Srinivasan, P.C., 2013. The role of inflammatory cytokines and the RANKL-RANK-OPG molecular triad in periodontal bone loss-A review. Clin. Cellular Immunol., S13: 007-007. DOI: $10.4172 / 2155-9899 . S 13-007$

Taylor, J.J., P.M. Preshaw and E. Lalla, 2013. A review of the evidence for pathogenic mechanisms that may link periodontitis and diabetes. J. Clin. Periodontol., 40: S113-S134. DOI: 10.1111/jcpe.12059

Tuter, G., M. Serdar, B. Kurtis, S.G. Walker and A. Atak et al., 2010. Effects of scaling and root planing and subantimicrobial dose doxycycline on gingival crevicular fluid levels of matrix metalloproteinase-8, -13 and serum levels of HsCRP in patients with chronic periodontitis. J. Periodontol., 81: 1132-1139. DOI: 10.1902/jop.2010.090694

Vernal, R., J. Diaz-Zúñiga, S. Melgar-Rodríguez, M. Pujol, E. Diaz-Guerra et al., 2014. Activation of RANKLinduced osteoclasts and memory $\mathrm{T}$ lymphocytes by Porphyromonas gigingivalis is serotype dependant. $\mathrm{J}$. Clin. Periodontol., 41: 451-459.

DOI: $10.1111 /$ jcpe. 12236

$\mathrm{Xu}$, J., H.F. Wu, E.S.M. Ang, K. Yip and M. Woloszyn et al., 2009. NF-kB modulators in osteolytic bone diseases. Cytokine Growth Factor Rev., 20: 7-17. DOI: 10.1016/j.cytogfr.2008.11.007 
Wara-Aswapati, N., R. Surarit, A. Chayasadom, J.A. Boch and W. Pitiphat, 2007. RANKL upregulation associated with periodontitis and porphyromonas gingivalis. J. Periodontol., 78: 1062-1069. DOI: 10.1902 /jop.2007.060398

Williams, R.C. and S. Offenbacher, 2000. Periodontal medicine: The emergence of a new branch of periodontology. Periodontology, 23: 9-12.

DOI: $10.1034 / \mathrm{j} .1600-0757.2000 .2230101 . x$

Yokoyama, M., T. Ukai, E.R. Ayon Haro, T. Kishimoto and Y. Yoshinaga et al., 2011. Membrane-bound CD40 ligand on $\mathrm{T}$ cells from mice injected with lipopolysaccharide-induced osteoclastogenesis. J. Periodontal Res., 46: 464-474.

DOI: $10.1111 /$ j.1600-0765.2011.01362.x
Zhao, L., Y. Zhou, Y. Xu, Y. Sun and L. Li et al., 2011. Effect of non-surgical periodontal therapy on the levels of Th17/Th1/Th2 cytokines and their transcription factors in Chinese chronic periodontitis patients. J. Clin. Periodontol., 38: 509-516. DOI: $10.1111 / \mathrm{j} .1600-051 X .2011 .01712 . \mathrm{X}$ 